\title{
Effect of Reduced $\boldsymbol{k}_{0}$ Zone on Time-Dependent Analysis of Tunnels
}

\author{
Mohammed Y. Fattah, Kais T. Shlash, and Nahla M. Salim \\ Building and Construction Engineering Department, University of Technology, Baghdad, Iraq \\ Correspondence should be addressed to Mohammed Y. Fattah, myf_1968@yahoo.com
}

Received 28 July 2010; Revised 29 November 2010; Accepted 27 March 2011

Academic Editor: Manolis Papadrakakis

Copyright () 2011 Mohammed Y. Fattah et al. This is an open access article distributed under the Creative Commons Attribution License, which permits unrestricted use, distribution, and reproduction in any medium, provided the original work is properly cited.

\begin{abstract}
The problem of the proposed "Baghdad metro line" which consists of two routes of $32 \mathrm{~km}$ long and 36 stations is analyzed. The tunnel is circular in cross-section with a $5.9 \mathrm{~m}$ outer diameter. The finite element analyses were carried out using elastic-plastic and modified Cam clay models for the soil. The excavation has been used together with transient effects through a fully coupled Biot formulation. All these models and the excavation technique together with Biot consolidation are implemented into finite-element computer program named "Modf-CRISP" developed for the purpose of these analyses. The results indicate that there is an inward movement at the crown and this movement is restricted to four and half tunnel diameters. A limited movement can be noticed at spring line which reaches $0.05 \%$ of tunnel diameter, while there is a heave at the region below the invert, which reaches its maximum value of about $0.14 \%$ of the diameter and is also restricted to a region extending to 1.5 diameters. The effect of using reduced $k_{0}$ zone on excess pore water pressure and surface settlement (vertical and horizontal) was also considered and it was found that the excess pore water pressure increases while the settlement trough becomes deeper and narrower using reduced $k_{0}$.
\end{abstract}

\section{Introduction}

Anyone who sets out to construct underground works finds themselves having to tackle and solve a particularly complex civil engineering problem, because it is far more difficult to determine the basic design specifications for underground works in advance than it is for surface constructions. It is not, as with surface constructions, a question of gradually assembling materials (steel, reinforced concrete, etc.) with well-known strength and deformation properties to build a structure which, when subject to predictable loads, finds its future equilibrium in the desired final configuration. On the contrary, one has to intervene in a preexisting equilibrium and proceed in some way to a "planned disturbance" of it in conditions that are only known approximately.

Three characteristic zones can be identified during tunnel advance in an unlined tunnel:

(1) an undisturbed zone, where the soil or rock mass is not yet affected by the passage of the face;

(2) a tunnel face or transition zone, corresponding to the radius of influence of the face, in which its presence has a considerable effect;
(3) a stabilisation zone, where the face no longer has any influence and the situation tends to stabilise (if possible).

It is important to observe that, in passing from the undisturbed zone to the stabilisation zone, the medium passes from a triaxial to a plane stress state and that the face zone is where this transition takes place. Consequently, this is the most important zone for the design engineer. It is here that the action of excavation disturbs the medium and it is on this zone that all the attention of the design engineer must be focused for proper study of a tunnel. It is not possible to achieve this without employing three-dimensional analysis approaches [1].

\section{Previous Studies on Tunneling Settlements}

2.1. Closed-Form Solutions. This type of analysis is based on "closed-form" solution to the problem of an isotropically stressed opening in homogeneous medium. It can help designers and engineers as a rough approximation for deformation and stress development estimates. A major use of the method is as a quick first parametric analysis for 
several parameters such as the elastic or strength properties. The limitations of the method are also the assumptions behind it [2].

(i) The tunnel is circular having a radius $R$.

(ii) Plane strain conditions apply to the problem.

(iii) Medium is elastic and perfectly plastic.

Davis et al. [3] presented lower and upper bound analytical solutions for the collapse load of shallow tunnel with support pressure in cohesive soil. They used the case of plane strain unlined circular tunnel (radial ground movements), a plane strain heading face (movements), and a circular tunnel heading (full three-dimensional case). The difference between the lower and upper bound collapse loads was the greatest (with almost a factor of two between them) for the three-dimensional case, indicating that there is a difficulty in applying analytical solution in three dimensions.

Data from centrifuge modeling [4] confirmed the solution for a plane strain unlined circular heading with the best agreement for $C / D$ less than 3 ( $C$ is the depth of the tunnel center and $D$ is its diameter). The value of $C / D$ has since become established as a practical transition between shallow tunnels, where the failure mechanics involves the ground surface, and deep tunnels where the failure mechanism is symmetric about the tunnel axis and the surface has little influence [5].

Sagaseta [6] proposed closed form solutions for obtaining the strain field in incompressible soil by combining fluid flow with elastic solutions. Chow [7] used Sagaseta approach to derive the solution for vertical displacement at the soil surface as follows:

$$
\delta=-\frac{\gamma D^{2} Z^{2}}{4 G\left(X^{2}+Z^{2}\right)},
$$

where $\gamma$ is the unit weight of the soil, $Z$ is the depth of the tunnel's center, and $G$ is the shear modulus of the soil.

Carter [8] presented a semianalytical solution for dissipation of pore pressure around a vertical hole in a hydrostatic stress field. An isotropic linear elastic, perfectly plastic porous medium with incompressible constituents is used. It was shown that the yielding of the ground simultaneously induces excess negative pore pressure.

Mair and Taylor [9] studied soil plasticity by comparing subsurface ground movements around tunnels in London clay with closed-form solutions for contracting spherical and cylindrical cavities in a linear elastic perfectly plastic medium, respectively. These are

$$
\begin{gathered}
\frac{\delta}{D}=\frac{S_{u}}{3 G}\left(\frac{D}{r}\right)^{2} e^{(0.75 r-1)}, \\
\frac{\delta}{D}=\frac{S_{u}}{2 G}\left(\frac{D}{r}\right) e^{(N-1)},
\end{gathered}
$$

where $\delta$ is the radial movement at radius $r, S_{u}$ is the undrained shear strength of soil, $N$ is the $\sigma_{v} / S_{u}$ the stability ratio, $\sigma_{v}$ is the overburden stress at the center of the tunnel before excavation starts, and $G$ is the shear modulus of soil.
The axial displacements ahead of tunnels are shown to vary with $(D / r)^{2}$ (i.e., the spherical cavity solution), while the radial movements around tunnels appear to follow variation in $(D / r)$ (i.e., the cylindrical cavity solution).

These analyses were extended to include a permanent tunnel lining which prevents the cavity from fully unloading to zero stress modifying (3) to be

$$
\frac{\delta}{D}=\frac{S_{u}}{2 G}\left(\frac{D}{r}\right) e^{(N(1-n)-1)},
$$

where $n$ is the proportion of the overburden pressure taken by the lining.

Verruijt and Booker [10] modified the elastic solution of Sagaseta [6] by applying various values of Poisson's ratio and included the ovalization of the tunnel opening. The solutions yielded slightly wider profile of lateral deformation induced by tunneling with tunnel boring machines.

Chou and Bobet [11] developed an analytical solution for shallow tunnels in saturated ground. Plane strain linear elastic soil model was used for the ground and the liner. Deformations and stresses were obtained for short- and long-term conditions. A comparison was also made between predictions and observations, and a good agreement was obtained and generally within 15\% difference. The conclusions drawn are as follows.

(i) Most of the ground movements are caused by the gap parameter, which is a measure of the threedimensional deformations at the tunnel face, the physical gap between the liner and the perimeter of the excavation, and of the workmanship.

(ii) Most of the ground deformations take place within a distance of three to four radii around the tunnel.

Some of their results from Green Park underground tunnel are shown in Figure 1.

2.2. Empirical Solutions. The transverse profile of vertical settlement due to tunnel in undrained condition follows a Gaussian (or normal) distribution curve as shown in Figure 1(a). This curve was first proposed by Peck [12] who compared it with many sets of field data from tunneling. Rankin [13] backed up Peck's assertion by examinations of subsequent field data. The formula which is used by Peck is as follows:

$$
\delta=\delta_{\max } e^{\left(-x^{2} / 2 i^{2}\right)}
$$

where $\delta_{\max }$ is the maximum vertical settlement over the axis of the tunnel, $x$ is the transverse distance from the axis to the point of interest, and $i$ is the width of the settlement trough which is dependent on the ground conditions and not linked to the tunneling method.

\section{Definition of the Problem}

It can be concluded from previous studies that only elastic solutions have been derived and published up to now, concerning the soil deformation and surface settlements 


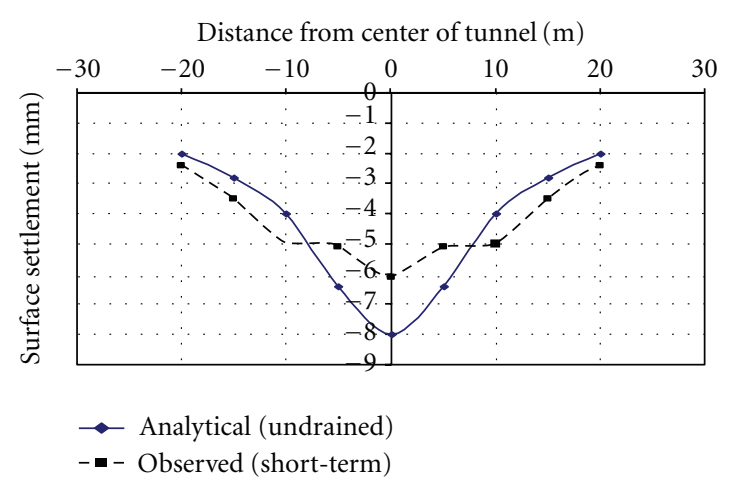

(a)

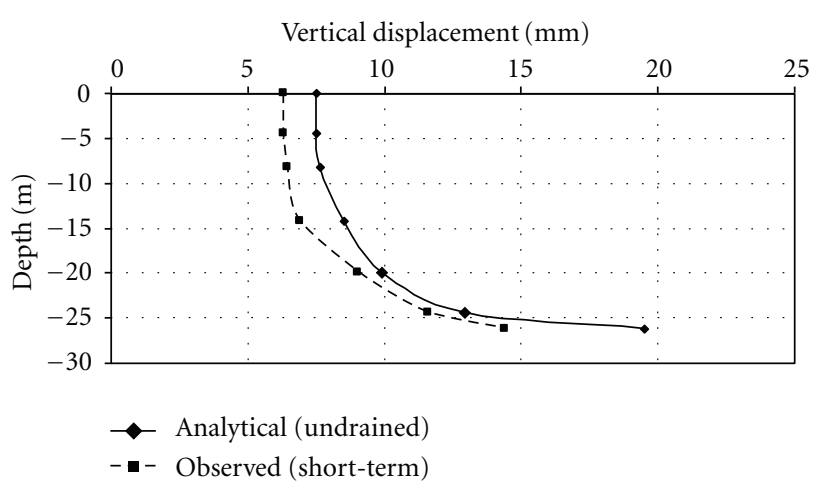

(b)

FIGURE 1: Green park underground tunnel: (a) surface settlement; (b) subsurface settlement (after [11]).

development, for the shallow tunnel case (elastic solutions in half-space).

In the present study, the problem represents "Baghdad metro line" which consists of two routes of $32 \mathrm{~km}$ long and 36 stations. The tunnel is circular in cross-section with a $5.9 \mathrm{~m}$ outer diameter and $0.45 \mathrm{~m}$ of concrete lining thickness. The geological section for a specified position with tunnel axis at a depth of $18 \mathrm{~m}$ is shown in Figure 2. In this analysis, it is required to study the temporary support pressure during the tunnel excavation. Therefore, during this stage, the final concrete lining is not simulated.

\section{Boundary Conditions of Numerical Analysis and Material Parameters}

The material parameters used in the analysis of Baghdad metro are given in Table 1. Figure 3 shows the finite element mesh used in the numerical analyses and the following supposed conditions are used.

(1) The boundary condition is that there is only horizontal restriction on the left and right vertical sides, and there are both horizontal and vertical restrictions at the bottom boundary.

(2) The problem boundaries are assumed to be impermeable on both vertical sides in addition to the bottom boundary. The water table is at the ground surface.

Eight-node isoparametric elements are used to model the soil and concrete lining. Four-node elements are used for modeling pore water pressure. Symmetry is assumed along the tunnel vertical axis containing the crown, spring line, and invert. This situation makes it possible to mesh just one half of the entire geometry as shown in Figure 3. The excavation process is assumed to occur under plane strain conditions since the effect of advancing the tunnel at the face is not considered in this analysis. In linear elastic analysis, the excavation process can be applied in a single instantaneous step. In nonlinear stress-strain relationship, the program CRISP allows the effect of element removal to be spread over several increments in an "increment block." Element stiffness

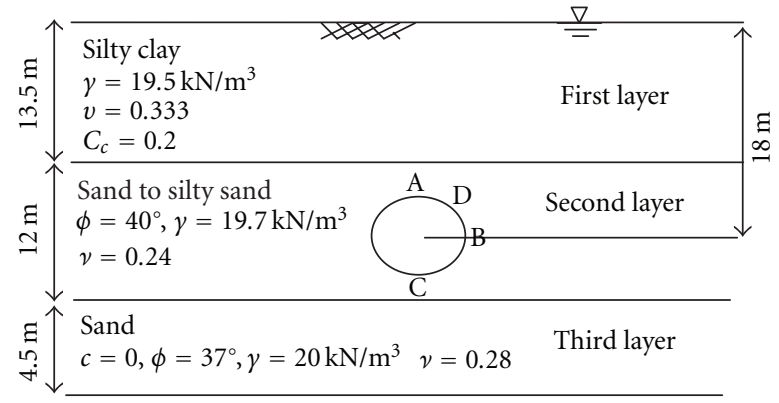

Figure 2: Geological profile and soil material properties along Baghdad metro line.

is always removed in the first increment of a block, but the associated loads are distributed over all the increments in the block. The excavation process was assumed to last for two hours per meter length, and, in nonlinear analysis, it was spread over fifty increments.

CRISP adopts Biot's theory of consolidation which assumes that the total stresses are dependent upon the excess pore pressure. The finite element formulation of Biot's theory facilitates the application of the theory to different problems subjected to different boundary conditions. This formulation may also be used for inhomogeneity and anisotropy and processes to evaluate the time rate of settlement for different two- and three-dimensional problems.

Due to circular geometry of the tunnel, it is suitable to use TBM machine. Therefore, the design values of the machine's support pressure must be calculated.

In the context of this paper, the excavation boundary condition is needed for the modeling of tunnel. CRISP allows elements to be removed to simulate excavation. The implied loading for this case is automatically calculated by the program and will be explained as follows. When a tunnel is excavated, unloading and stiffness reduction occur simultaneously. The first results from removal of material having self-weight. The second occurs because the ground structure is altered. To model the first of these processes, loads are applied to the mesh remaining after excavation. The 


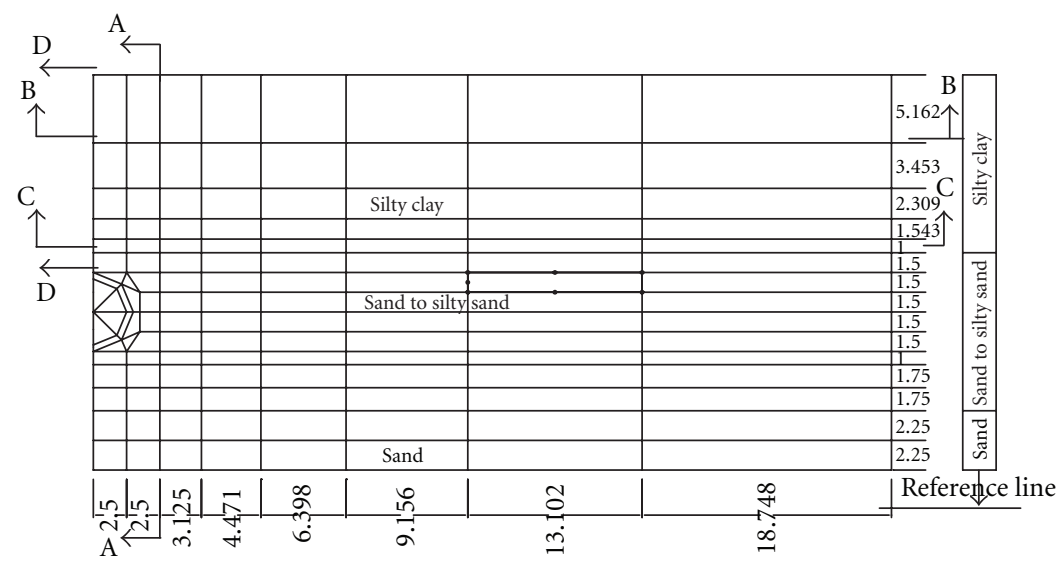

Figure 3: The finite element mesh for the problem.

TABLE 1: The soil parameters of the analyzed section of the proposed Baghdad metro line.

\begin{tabular}{lcccc}
\hline Type of soil & Modulus of elasticity $(E)(\mathrm{kPa})$ & Poisson's ratio $(\nu)$ & Unit weight $(\gamma)\left(\mathrm{kN} / \mathrm{m}^{3}\right)$ & Permeability $k x=k y(\mathrm{~m} /$ day $)$ \\
\hline Silty clay & 80000 & 0.333 & 19.5 & $8.64 \times 10^{-6}$ \\
Sandy to silty sand & 180000 & 0.263 & 19.7 & Drained \\
Sand & 150000 & 0.284 & 20.0 & Drained \\
\hline
\end{tabular}

second process is modeled by excluding the stiffness of each element excavated from the calculation of structure stiffness.

The first component of excavation requires the calculation of nodal loads equivalent to self-weight, or body force, associated with the volume of material represented by each element. In calculating these loads, the nodal forces due to self-weight are calculated from the following equation:

$$
F_{g}=\int_{\nu} N^{T} \gamma d \nu
$$

where $N$ is the matrix of element shape functions.

Secondly the loads $F_{i}$ associated with nodes on the tunnel periphery, equivalent to the internal stress, $\sigma_{i}$ are calculated using

$$
F_{i}=\int_{\nu} B^{T} \sigma_{i} d \nu
$$

where $v$ is the volume of complete, unexcavated mesh and $B$ is the strain-displacement matrix.

The third load is the pressure load along element boundary which is equal to

$$
F_{b}=\int_{S} N^{T} \tau d \text { (area). }
$$

So the model loads for the removed elements are given by

$$
F=\int_{\nu} B^{T} \sigma_{i} d \nu-\int_{\nu} N^{T} \gamma d \nu-\int_{S} N^{T} \tau d(\text { area })
$$

where the first term represents the loads equivalent to in situ stresses.
In the present study some modifications are added to the main finite element computer program (CRISP) to obtain the present computer program (Modf-CRISP). The program has been designed so that new element types can be added in a straightforward manner. In the present work, eightnode quadrilateral isoparametric elements are added. The element incorporates three degrees of freedom per node, two degrees of freedom for displacement with excess pore water pressure degree of freedom for consolidation analysis. A new soil model has also been added to CRISP. The model is following Mohr-Coulomb failure criterion according to the steps directed by Britto and Gunn [15]. Two-dimensional mesh generation was carried out by developing a program named MG2D, this program was written in FORTRAN 77 and it can arrange all data related to element and its properties in a way that can be used directly by CRISP program.

\section{Points of Interest When Analyzing a Tunnel}

Figure 2 shows the location of points to be focused upon in the analysis.

(1) The tunnel crown is the topmost point of the tunnel section (point A).

(2) The spring line refers to point located at the side wall of the tunnel (point B).

(3) The invert refers to the bottom of the tunnel section (point C).

(4) The shoulder is referred to by point (D). 


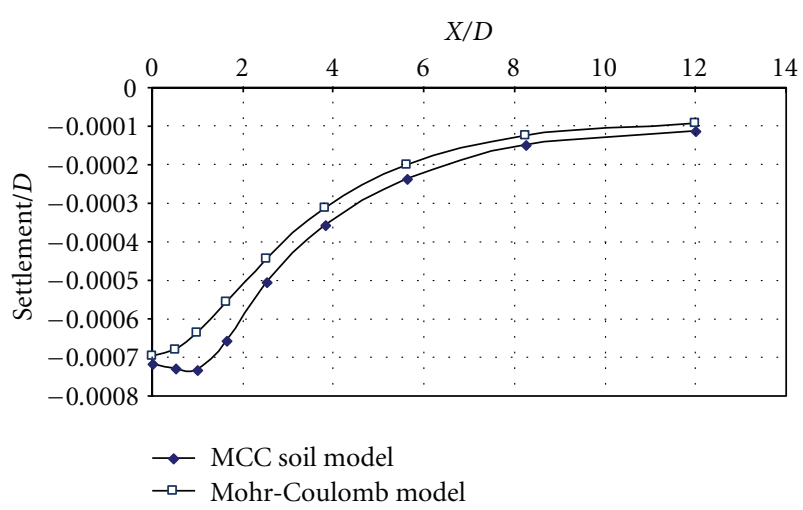

Figure 4: Soil surface subsidence using different soil models.

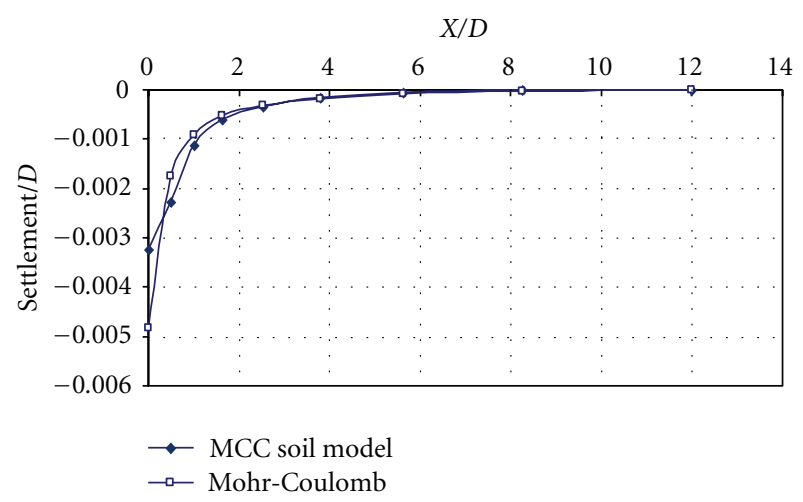

Figure 5: Comparison between settlements above the crown along section C-C using two soil models.

\section{Undrained Analyses}

Two kinds of analyses are carried out:

(a) using elastic-plastic constitutive model for all soil layers,

(b) using modified Cam clay model (MCC) for the first soil layer (upper layer) and elastic-plastic Mohr Coulomb for the other two layers.

The silty clay parameters used with MCC soil model are presented in Tables 1 and 2. The parameters are calculated depending on the data of NCCLR [16].

6.1. Surface Settlement due to Tunneling. The magnitude of movements generally depends on the soil type, ground water condition, construction method, tunnel dimensions, and location. Ground movements around tunnels lead to surface subsidence.

Tunnel construction inevitably leads to displacements in the soil, and these may lead to damage to nearby structures. These ground movements are associated with two mechanisms:

(a) radial movements of the soil as a result of cutting during tunnel construction,

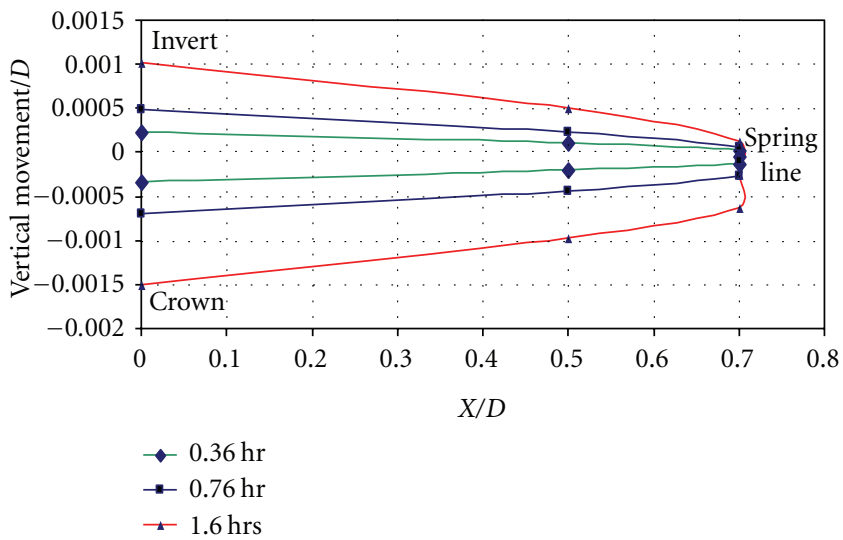

Figure 6: Vertical movement around the tunnel using MohrCoulomb failure criterion for the first layer.

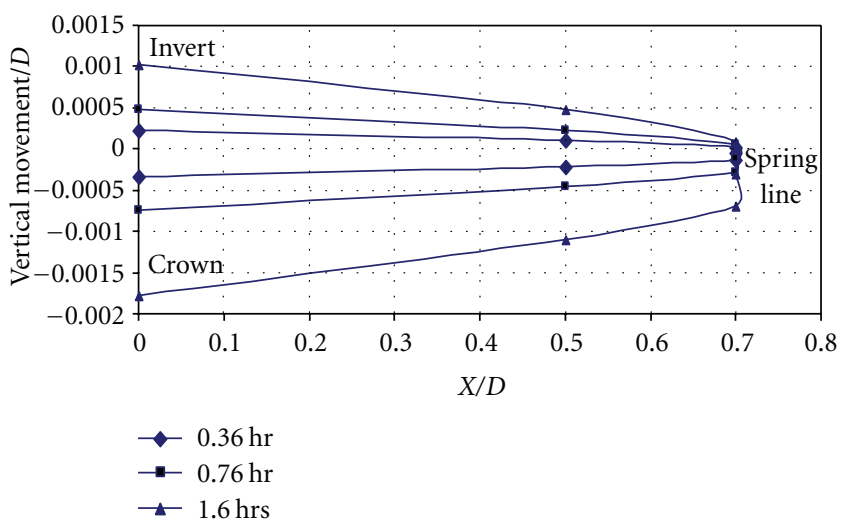

FIGURE 7: Vertical movement around the tunnel using MCC model for the first layer.

TABLE 2: Cam clay parameters for Baghdad silty clay soil.

\begin{tabular}{ccccc}
\hline$C_{c}$ & $C_{s}$ & $\lambda$ & $\kappa$ & $M$ \\
\hline 0.2 & 0.02 & 0.0866 & 0.00866 & 1.2 \\
\hline
\end{tabular}

Note. $\phi=30^{\circ} . C_{c}$ : the compression index, $C_{s}$ : the swelling index, $\lambda$ : the slope of the normal consolidation line, $\kappa$ : the slope of the swelling line, and $M$ : the gradient of the critical state line.

(b) ground movements associated with incomplete support of the tunnel face.

Figure 4 shows the variation of surface settlement due to tunneling using two different constitutive models: elasticplastic and modified Cam clay (MCC) for the first layer. In this figure, $x$ refers to the distance from tunnel axis to the end of the model. It can be seen that the maximum settlement using MCC for the first layer is $4.4 \mathrm{~mm}$, while the maximum settlement using Mohr Coulomb for the first layer is $4.2 \mathrm{~mm}$. This is due to lower value of plastic stiffness of the soil assumed by the MCC model which produces larger plastic strains. The settlement that is caused by the accumulation of the vertical displacement of all the elements above the tunnel is also greater in the case of the MCC model. From the results, 


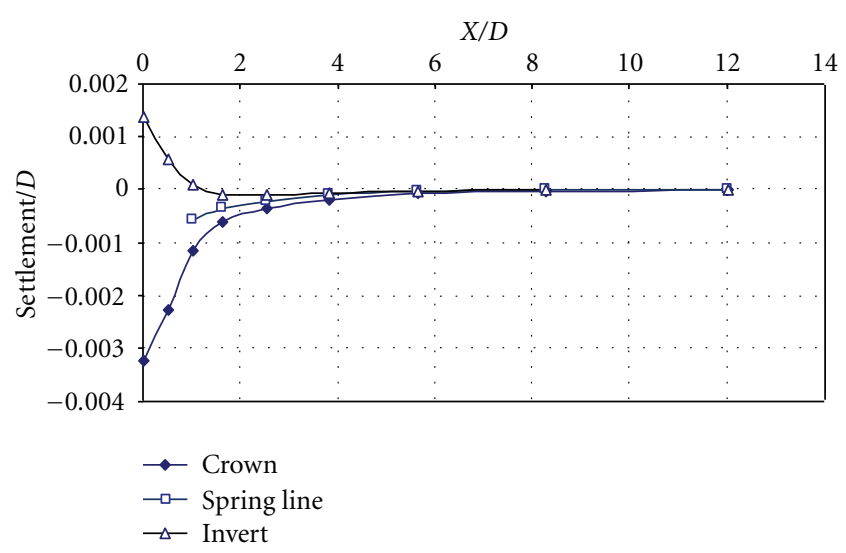

Figure 8: Vertical displacement at the end of excavation of the tunnel using MCC model.

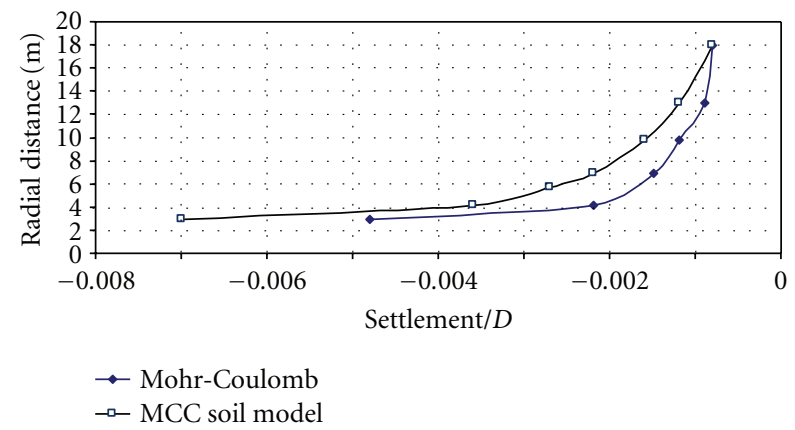

Figure 9: Variation of the vertical settlement along the radial distance along section D-D above the crown.

it can be seen that the constitutive models of the first layer have small effect on the settlement above the crown.

Figure 5 shows that the dimensionless settlement above the crown using Mohr-Coulomb is about $0.17 \%$ greater than the dimensionless settlement using MCC constitutive model. It is also shown that most deformations occur near the tunnel opening and they are largely restricted to a zone within twice tunnel diameters.

It can also be concluded from Figures 4 and 5 that the two constitutive relations, Mohr-Coulomb and MCC, give approximately similar displacements when the distance from center of the tunnel increases.

Figure 6 shows the variation of vertical movement at different points around the tunnel during excavation (which was assumed to last 2 hours). In this figure, negative movement means an inward movement towards the tunnel's center while a positive value of vertical movement means an upward movement towards the tunnel center. There is an inward movement at the crown and all points above the center of the tunnel, while there is an upward movement at the invert and all points below the center line of the tunnel. This means that there is a heave taking place at all points below the center line of the tunnel. At the spring line, very small movement of about $0.05 \%$ of the diameter occurs, while the inward movement may reach $0.15 \%$ of the diameter at the crown and a maximum outward movement reaches $0.1 \%$ of the diameter at the invert. This difference is due to relatively high in situ stresses at the invert and low soil strength and usually the invert is not allowed to have perfect arch for stress redistribution [17].

The same results are obtained in Figure 7 using MCC constitutive model, except that the maximum settlement at the crown reaches approximately $0.175 \%$ of the tunnel diameter.

\section{Movement of the Soil around the Tunnel}

The movements of the soil around the tunnel at the end of excavation at typical points (crown, spring line, and the invert) are shown in Figure 8. The results indicate that there is an inward movement at the crown and this movement is restricted to four and half tunnel diameters. A limited movement can be noticed at the spring line which reaches $0.05 \%$ of tunnel diameter, while there is a heave at the region below the invert, which reaches its maximum value of about $0.14 \%$ of the diameter and is also restricted to a region extending to 1.5 diameters.

Figure 9 indicates the dimensionless settlement along section D-D (shown in Figure 3), the radial distance above the crown, using two soil constitutive models. The results show that the maximum settlement reaches about $0.49 \%$ of the diameter near the center of the tunnel and is reduced to $0.08 \%$ of the diameter at the surface using Mohr-Coulomb soil model, while it reaches $0.7 \%$ of the tunnel diameter near the center of the tunnel and is reduced to $0.08 \%$ at the surface using MCC soil model.

\section{Stress Changes around the Tunnel}

Typical effective stress time history around the tunnel opening obtained by the finite element analysis is shown in Figure 10. The stresses are normalized by division of each stress by the initial overburden pressure. At the crown, excavation induces a reduction in the radial stress $\left(\sigma_{v}^{\prime}\right)$ and a small increase in the tangential stress $\left(\sigma_{h}^{\prime}\right)$ as shown in Figures 10(a) and 10(b). However, the reduction of radial stress is rapid, and this causes the radial stress to become the minor principal stress $\left(\sigma_{3}\right)$ and the tangential stress to become the major principal stress $\left(\sigma_{1}\right)$. This means that the principal stresses rotate by $90^{\circ}$ and $\sigma_{1}$ remains horizontal after rotation. At the spring line, excavation induces a reduction in the radial stress $\left(\sigma_{h}^{\prime}\right)$ to become $\left(\sigma_{3}\right)$ and an increase in the tangential stress to become $\left(\sigma_{1}\right)$. This means that the principal stresses do not change at the spring line as shown in Figure 10(b). These results agree with the findings of Lee and Rowe [18].

From this, the conclusion drawn is that the stress above and below the tunnel corresponds approximately to triaxial extension and that the strength and deformation profile in these regions can be estimated from triaxial extension tests, while, near the spring line of the tunnel, the stress is intermediate between the triaxial compression and extension. This was also suggested by Lo and Rowe [19] 


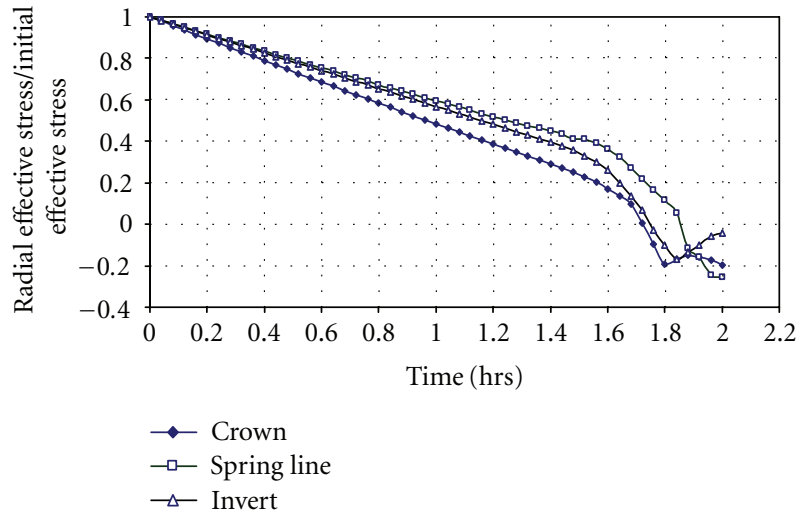

(a) Radial stresses

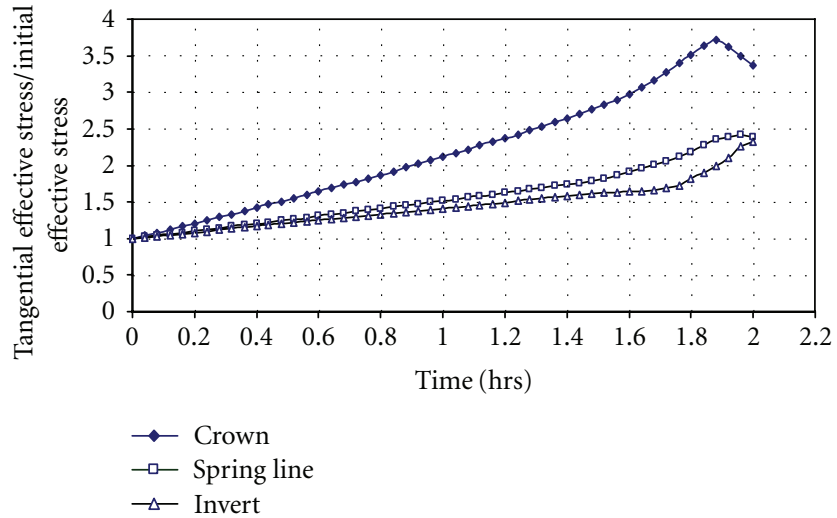

(b) Tangential stresses

FIGURE 10: Variation of stresses around the tunnel at the end of excavation.

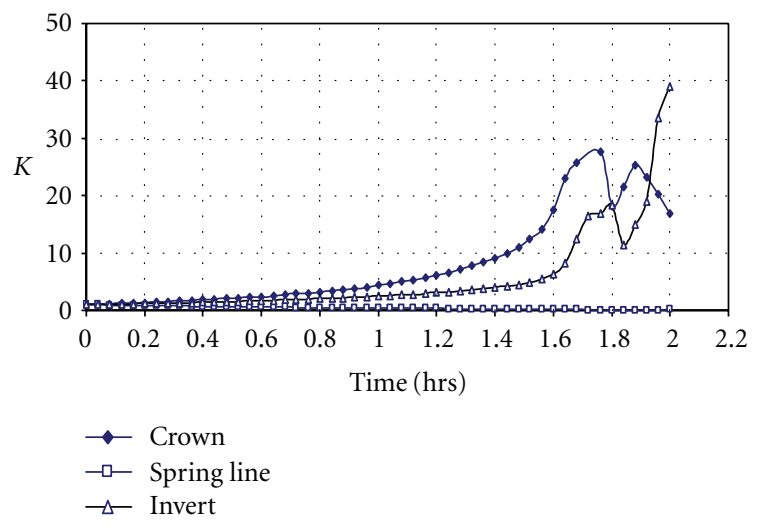

FIGURE 11: Variation of $K$ at the crown, spring line, and invert during the excavation of the tunnel.

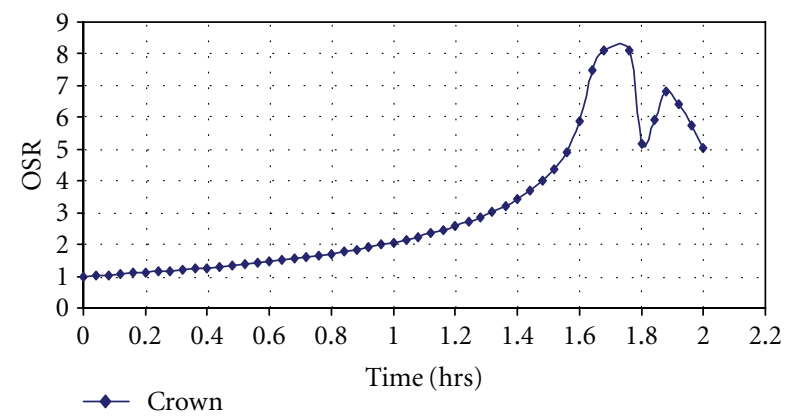

FIgURE 12: Variation of the overstress ratio (OSR) with time above the tunnel's crown.

and $\mathrm{Ng}$ and Lo [20]. The modulus and strength parameters in this region should therefore lie between the compression and extension values. For this reason, the modulus above the crown and below the invert is most critical.

The variation of the horizontal and vertical effective stresses around the tunnel can be studied by plotting the relation between $K$ which is the ratio of initial horizontal to vertical effective stresses and the excavation time which

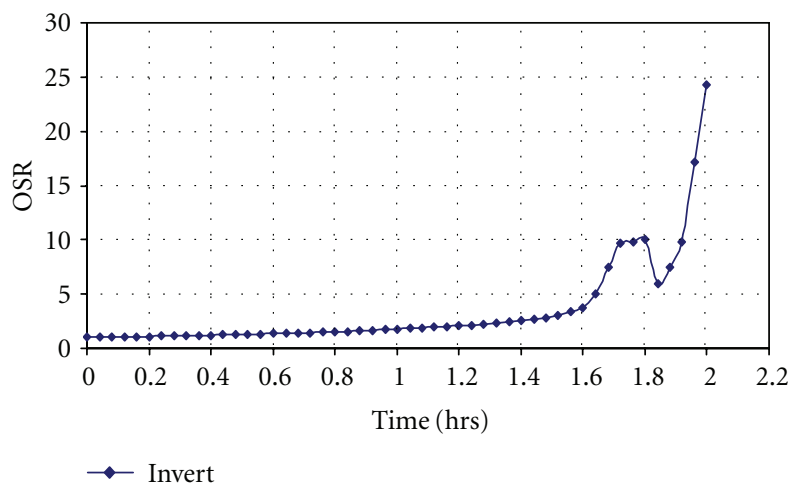

FIgURE 13: Variation of the overstress ratio (OSR) below the tunnel's invert.

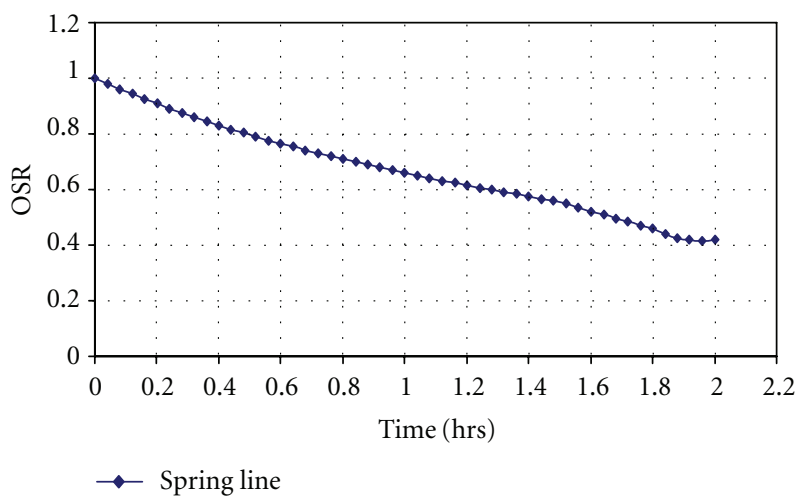

FIGURE 14: Variation of the overstress ratio OSR at the tunnel's spring line.

lasted two hours. Figure 11 shows that the value of $K$ remains constant and starts with initial value of (1) because $\sigma_{h}^{\prime}=\sigma_{v}^{\prime}$ (normally stressed sand). This value starts to increase and reaches the maximum value over a period of time which is approximately 1.67 hours.

Beyond this time, the value of $K$ decreases dramatically at the crown which means that the horizontal stress increases 


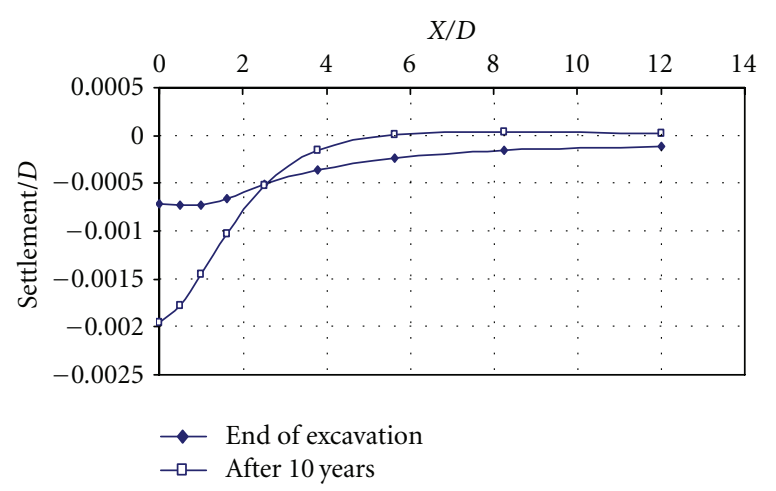

FIGURE 15: The surface settlement at the end of excavation and after consolidation using MCC soil model.

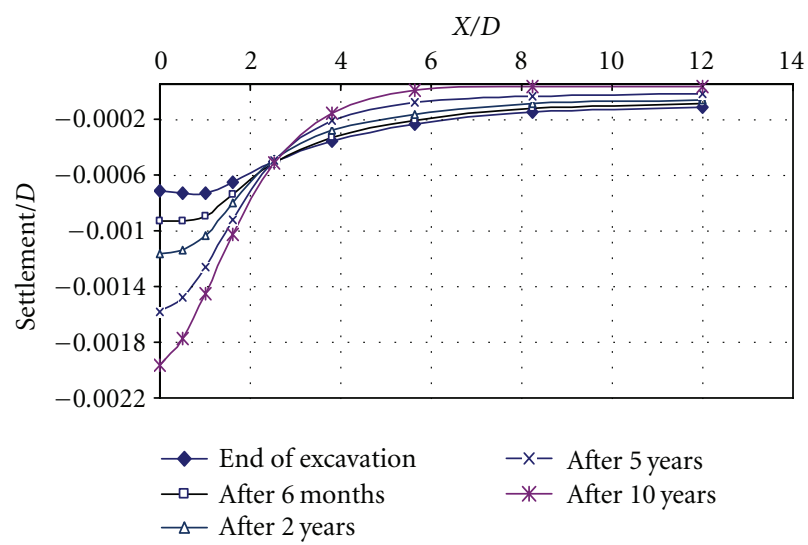

FIGURE 16: The surface settlement at different times using MCC soil model.

and the vertical stress decreases due to tunneling. This is clear in Figure 11. The value of $K$ at the crown reaches approximately 28; this is due to dramatic decrease in the vertical effective stress due to tunneling process, while, at the spring line, the variation is very small. It is expected that the soil at the crown transforms gradually to overstressed sand due to excavation of the tunnel. This is confirmed by the results shown in Figures 12, 13, and 14 which show the absolute value of the overstress ratio $\left(\mathrm{OSR}=\sigma_{o}^{\prime} / \sigma_{v}^{\prime}\right)$ changes with time.

Figure 12 shows that, at the crown, the OSR increases slowly at the start of excavation and then increases dramatically over a period of 1.67 hours. This increase may be due to a great reduction in the vertical effective stress. Then the value of OSR is reduced sharply and reaches about 5 at the end of excavation.

The OSR at the invert follows the same trend as shown in Figure 13 but it tends to increase and reach approximately 25 at the end of excavation. Both cases indicate that the soil changes from normal stress to overstress due to great reduction in the effective vertical stresses caused by tunneling while at spring line the OSR is reduced as the process of excavation progresses (Figure 14).

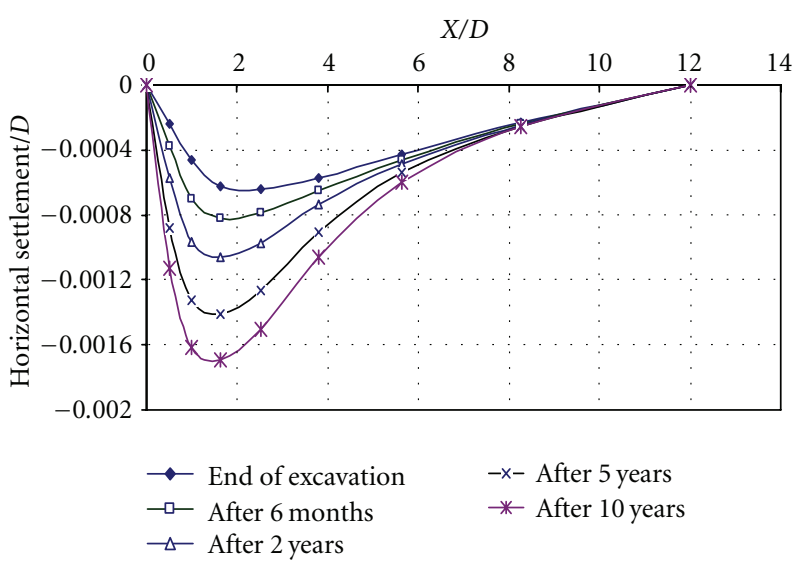

FIGURE 17: Surface horizontal displacement at the end of excavation and different times of consolidation using MCC soil model.

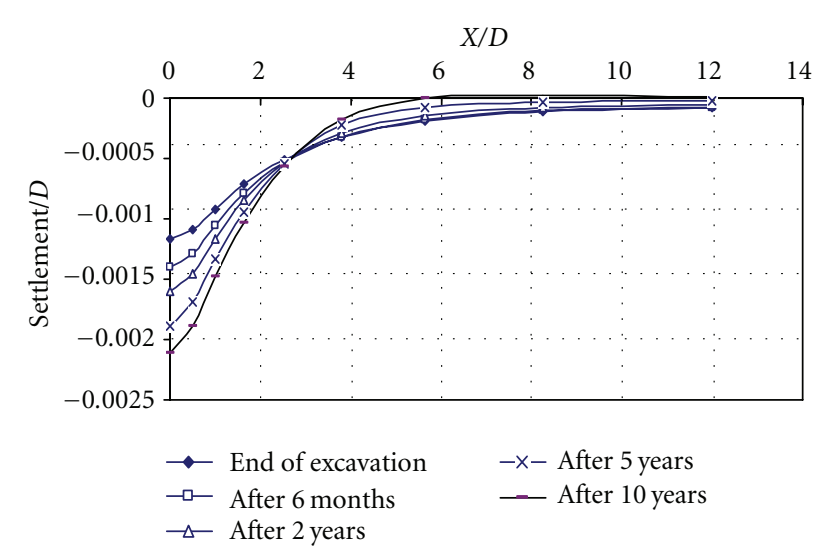

Figure 18: Variation of subsurface settlement along section B-B at different consolidation times using MCC soil model.

The comparison between the OSR at the crown, spring line, and the invert reveals that a great increase in the OSR occurs at the invert such that OSR at the invert is about 5 times that at the crown while there is a small reduction in OSR at the spring line due to continuous decrease in horizontal effective stress as a result of tunneling.

\section{Consolidation Analyses}

When a tunnel is excavated in a fully saturated soil, two types of time-dependent behaviors occur in the surrounding ground. The first is due to the intrinsic rate-dependent characteristics of the materials such as stress relaxation. The second is caused by movement of pore water due to the change of pore water pressure distribution in the surrounding ground. This section focuses on investigating the second type of time-dependent behavior of the surrounding ground.

The time-dependent behavior of tunnels can be modeled using a coupled consolidation finite element approach. In the following sections, the surface and subsurface settlements are studied using consolidation analyses for the first layer. The changes in excess pore water pressure are also considered. 


\section{Surface Settlement}

Figure 15 shows the normalized surface subsidence at the end of excavation and after 10-year consolidation. The maximum settlement reaches approximately $0.2 \%$ of the tunnel's diameter when consolidation analysis is considered while the maximum value approximately equals $0.07 \%$ of the tunnel's diameter at the end of excavation. This means that the maximum consolidation settlement above the tunnel is about three times that at the end of construction. This increase in consolidation settlement is due to dissipation of excess pore water pressure that causes an increase in effective stresses which leads to increase in vertical movement. The results also show that the consolidation process causes the settlement trough to be deeper and narrower and the affected region is reduced. A small heave can be noticed at the far end which is at a distance about 5.75 of the tunnel diameter. This may be due to the flow direction of strain vectors which move inward to the tunnel for the elements near the tunnel and move upward for the ground surface at the farther side.

The settlement trough also extends to five tunnel diameters using consolidation analysis while it is extended to twelve tunnel diameters using undrained analysis. In general, the settlement predicted from consolidation analysis is about twice the settlement using short term at the end of excavation. This result agrees with the results of Addenbrook [21].

Figure 16 shows the progress of surface settlement with time. The results show that, as the consolidation progresses, the settlement increases and the influenced region decreases. From Figure 16, the maximum consolidation settlement reaches approximately $0.2 \%$ of the tunnel diameter after 10 years while it reaches only $0.07 \%$ of the tunnel diameter at the end of excavation. In general, as the consolidation settlement progresses, the settlement trough gets deeper and narrower and the influenced region is reduced.

The progress of horizontal displacement with time is also studied. Figure 17 shows the horizontal displacement (normalized by the tunnel diameter) at different times. The results indicate that the maximum horizontal displacement occurs approximately at a distance of $2 \mathrm{D}$ at the end of excavation. This maximum horizontal displacement moves towards the tunnel opening as the consolidation progresses and it reaches its maximum value at a distance equal to $1.75 \mathrm{D}$ from the center of the tunnel after 10 years of consolidation.

\section{Subsurface Settlement}

In the previous section, the variation of the surface settlement with time due to consolidation is discussed. In this section, a subsurface settlement is studied with time due to consolidation. The settlement is investigated at two sections: section $\mathrm{B}-\mathrm{B}$ which is near the surface and about $5 \mathrm{~m}$ from the ground surface and section $\mathrm{C}-\mathrm{C}$ which is near the tunnel opening and approximately $13.5 \mathrm{~m}$ from the ground surface.

Figure 18 presents the variation of settlement along section (B-B) at different times. It can be seen that the subsurface settlement becomes deeper and the settlement

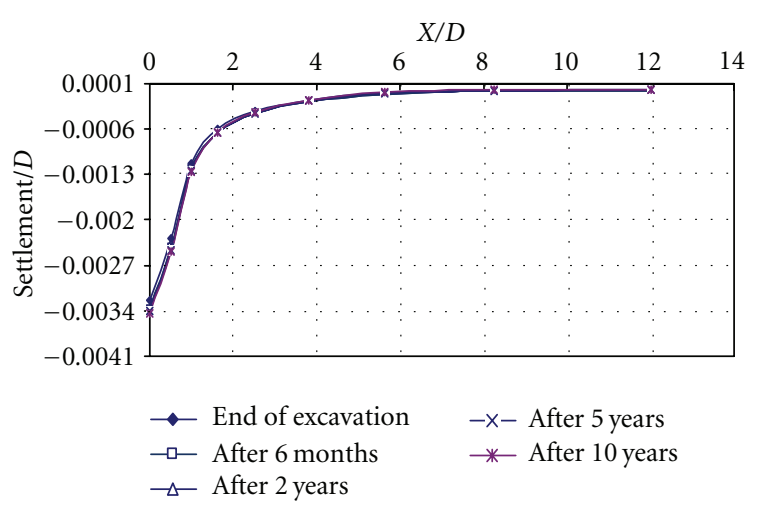

Figure 19: Subsurface settlement along section C-C at different consolidation times using MCC soil model.

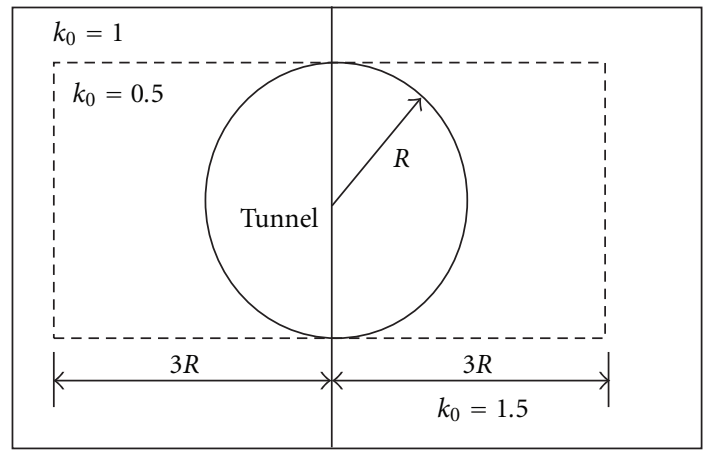

Figure 20: Layout of the zone of reduced $k_{0}$ (after [14]).

trough width becomes narrower with time. The maximum settlement is approximately equal to $0.12 \%$ of the tunnel diameter at the end of excavation and about $0.21 \%$ of the tunnel diameter after 10 years of consolidation.

At section $\mathrm{C}-\mathrm{C}$, the effect of consolidation is not clear and the maximum settlement reaches $0.33 \%$ of the tunnel diameter at the end of excavation and approximately $0.34 \%$ of the tunnel diameter after 10 years of consolidation as shown in Figure 19. This is due to the fact that section C-C lies on the border between silty clay layer and silty sand layer at which the dissipation of excess pore water pressure takes place within very short time and during the excavation stage.

\section{Reduced $\boldsymbol{k}_{0}$ Zone}

Addenbrook [21] compared the finite element settlement prediction of tunnel construction of Jubilee line in London for both $k_{0}=1.5$ and 0.5 with field measurements. The conclusion drawn is that the low $k_{0}$ cases show deeper and narrower settlement troughs and consequently are closer to the field data. Similar results are presented more recently by others for 2D and 3D analyses [22-24].

Addenbrook [21] performed axisymmetric analyses of a tunnel heading. The results show a reduction in radial stresses while hoop stresses around the tunnel boundary increase. It is concluded that, at tunnel spring line, the 


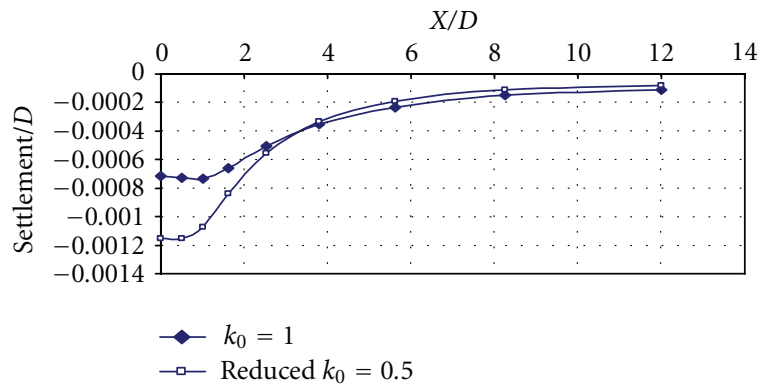

FIGURE 21: Variation of surface settlement depending on variation of $k_{0}$.

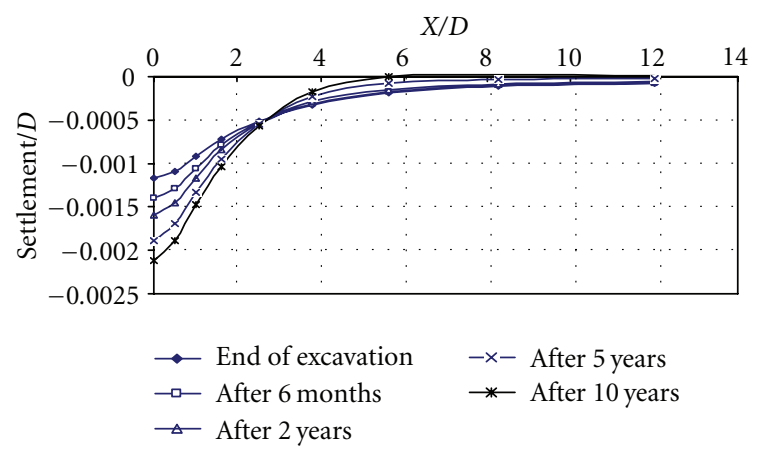

FIGURE 22: Variation of surface settlement at different times using reduced $k_{0}$ values.

reduction in radial stress causes the lateral stress ratio to reduce while it increases at the tunnel crown and invert.

This is also done in the present study. The change in stress state can be represented in a plane strain analysis by a zone of reduced $k_{0}$ around the tunnel. The plane of such zone is shown in Figure 20. Apart from this zone, the lateral earth pressure coefficient at rest was kept to a global value of $k_{0}=$ 1.0.

The results of such analysis are represented in Figures 21 and 22, which show the variation of surface settlement using $k_{0}=1.0$ and $k_{0}=0.5$, respectively. The analysis using $k_{0}=$ 0.5 shows deeper and narrower settlement trough than the analysis using $k_{0}=1.0$. This may be due to the direction of the major principal stress which is in the vertical direction when $k_{0}$ equals 1.0 .

This was also found by Addenbrooke et al. [25] whose analyses were carried out by reducing $k_{0}$ from 1.5 to 0.5 around the tunnel zone.

From the results, a conclusion can be drawn that, as the time of consolidation progresses, the effect of reduced $k_{0}$ is clearer. The maximum settlement at the end of excavation reaches about $0.07 \%$ of the tunnel diameter using $k_{0}=1.0$ while it reaches $0.12 \%$ using $k_{0}=0.5$.

Comparing Figures 16 and 22 using two different $K_{0}$ values, the results reveal that the surface settlement at the end of consolidation reaches about $0.20 \%$ of the tunnel diameter using $k_{0}=1.0$, while it reaches about $0.22 \%$ of the tunnel diameter using reduced $k_{0}=0.5$. This is due to increase

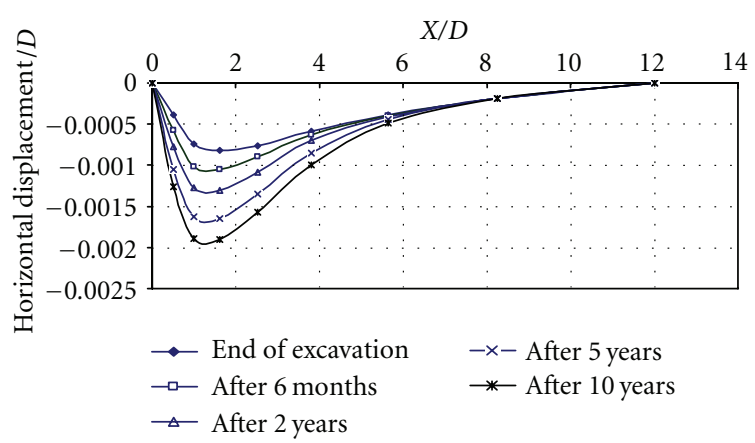

Figure 23: The surface horizontal displacement using reduced $k_{0}$ values.

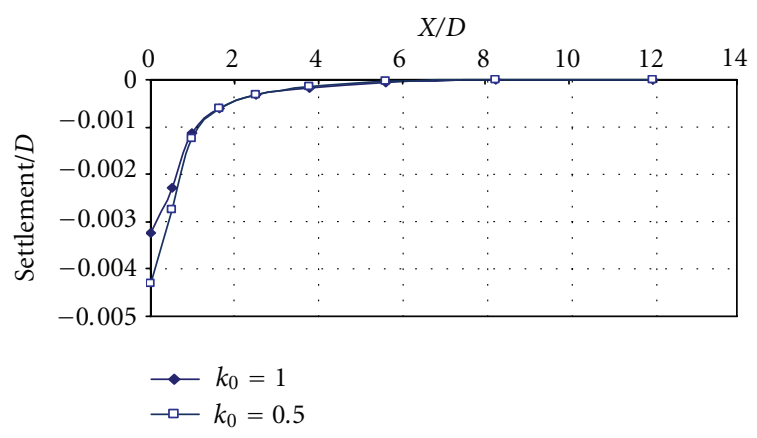

FIGURE 24: Variation of the subsurface settlement along section C-C using different $k_{0}$ values.

in the vertical effective stress at reduced $k_{0}$ which causes increase in the vertical movement.

Figure 23 shows the effect of using a zone of reduced $k_{0}\left(k_{0}=0.5\right)$ on the surface horizontal displacement. It can be noticed that the reduction of $k_{0}$ causes an increase in horizontal displacement. The maximum displacement at the end of excavation is approximately $0.08 \%$ of the tunnel diameter, and this increase is due to consolidation. It reaches a value approximately equal to $0.19 \%$ of the tunnel diameter after 10 years of consolidation.

Compared with Figure 17, the effect of using reduced $k_{0}$ is clear; the maximum displacement reaches about $0.07 \%$ of the tunnel diameter at the end of excavation while it reaches about $0.19 \%$ of the diameter after 10 years of consolidation. The width of the settlement trough is also reduced using a zone of reduced $k_{0}$.

So in general, using a zone of reduced $k_{0}$ causes an increase in the vertical displacement and reduction in the width of the settlement trough.

The effect of using reduced $k_{0}$ zone on the settlement above the crown at section C-C is clear in Figure 24. This effect is only limited to a region equal to the tunnel diameter. The maximum vertical movement using reduced $k_{0}$ is approximately equal to $0.43 \%$ of the tunnel diameter while it reaches $0.31 \%$ of tunnel diameter when using $k_{0}=$ 1.0. This difference is due to the increase in vertical stress which causes an increase in the vertical displacement. 


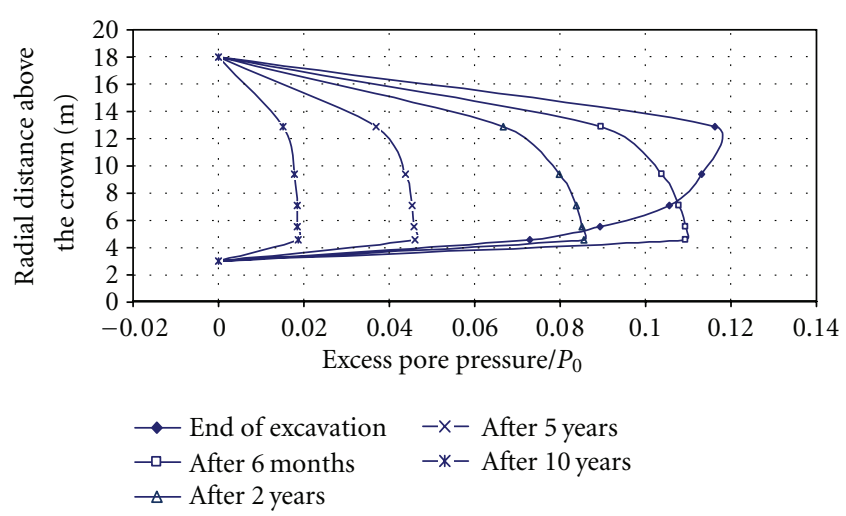

FIGURE 25: Variation of excess pore water pressure with time along section D-D using MCC soil model.

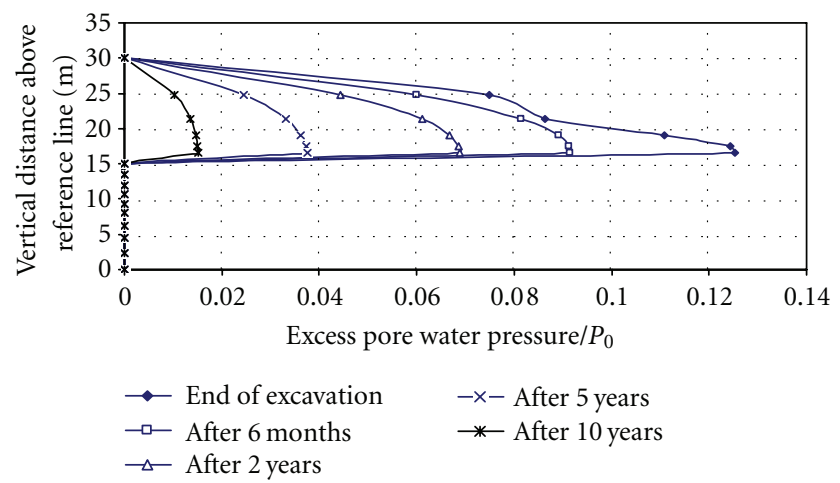

FIGURE 26: Variation of excess pore water pressure with time along section A-A using MCC soil model.

The evaluation of excess pore water pressure at the crown and at a radial distance from the tunnel center $r=3 \mathrm{~m}$ is shown in Figure 25. The results reveal that the maximum excess pore water pressure (normalized by dividing each value by the initial pore water pressure at the center line of the tunnel) occurs at a point $12 \mathrm{~m}$ above the center of the tunnel. Its value approximately equals $11.8 \%$ of the hydrostatic pore water pressure. The progress of consolidation causes dissipation of excess pore water pressure, and this leads to increase in vertical effective stress.

The vertical profile of excess pore water pressure along section A-A is shown in Figure 26. The results show that the maximum excess pore water pressure occurs approximately $4 \mathrm{~m}$ above the center of the tunnel, and, as the consolidation process progresses, the excess pore water pressure dissipates and reaches a minimum value that approximately equals $1.5 \%$ of the hydrostatic pressure after 10 years of consolidation.

The effect of using a zone of reduced $k_{0}$ on the distribution of excess pore water pressure is shown in Figure 27 which shows that the maximum excess pore water pressure occurs at a point $12 \mathrm{~m}$ above the center of the tunnel. Its value is approximately $13 \%$ of the hydrostatic pore water pressure. The effect of using a zone reduced $k_{0}$ causes an increase in the excess pore water pressure.

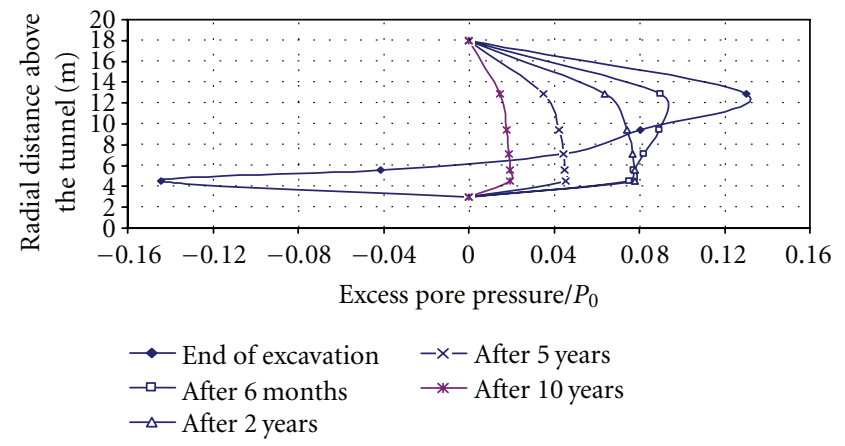

Figure 27: Variation of excess pore water pressure with time along section D-D above the crown using reduced $k_{0}$.

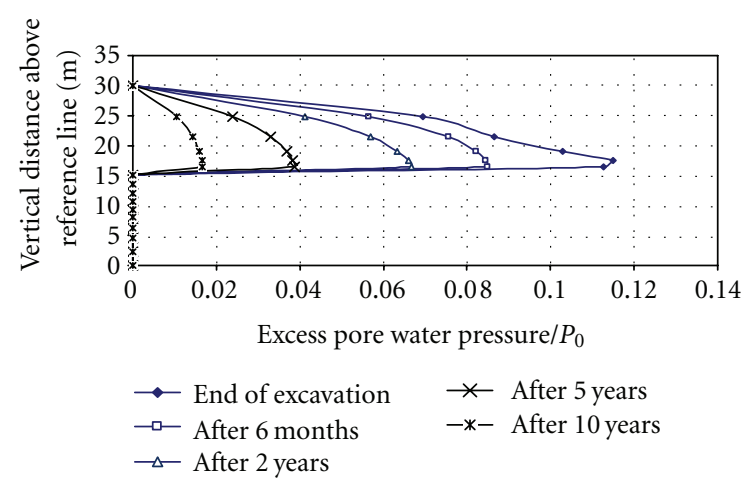

Figure 28: Variation of excess pore water pressure with time along section A-A using reduced $k_{0}$ values.

The variation of excess pore water pressure behind the tunnel is shown in Figure 28. The results show that the maximum positive excess pore water pressure reaches about $11.8 \%$ of the initial pore water pressure using reduced $K_{0}$. This value decreases to about $1.8 \%$ of the initial pore water pressure after 10 years of consolidation. Comparison of these results with those in Figure 25 shows that the maximum excess pore water pressure reaches approximately $12.5 \%$ at the end of excavation and about $1.75 \%$ of initial pore water pressure after 10 years of consolidation. This difference is a result of reducing $K_{0}$ which leads to increase in the effective vertical stress due to dissipation of excess pore water pressure.

\section{Conclusions}

(1) The modified Cam clay model revealed greater values of settlement in the soil than did the Mohr-Coulomb elastic-plastic model. This was attributed to the lower value of plastic stiffness of the soil assumed by the modified Cam clay model which produces larger plastic strains.

(2) Most deformations due to tunneling in clays occur near the tunnel opening and they are restricted to a zone within twice tunnel diameters. 
(3) All points above the tunnel showed downward movement while those below the tunnel were subjected to upper movement due to tunneling.

(4) At the crown of the tunnel, excavation induces a reduction in the radial stress $\left(\sigma_{v}^{\prime}\right)$ and a small increase in the tangential stress $\left(\sigma_{h}^{\prime}\right)$. The reduction of radial stress is rapid which causes that this stress becomes the minor principal stress $\left(\sigma_{3}\right)$ and the tangential stress becomes the major principal stress $\left(\sigma_{1}\right)$. This means that the principal stresses rotate by $90^{\circ}$ after excavation and $\left(\sigma_{1}\right)$ remains horizontal after rotation.

(5) The maximum consolidation settlement above the tunnel is about three times that at the end of excavation. The settlement trough extends only to 5 tunnel diameters using consolidation analysis while it is extended to 12 tunnel diameters using undrained analysis.

(6) Using a zone of reduced $k_{0}$ causes an increase in the vertical displacement, reduction in the width of the settlement trough, and increase in the excess pore water pressure. This effect is only limited to a region equal to the tunnel diameter. The maximum vertical movement using reduced $k_{0}$ is approximately equal to $0.43 \%$ of the tunnel diameter while it reaches $0.31 \%$ of tunnel diameter when using $k_{0}=1.0$. The effect of using a zone reduced $k_{0}$ causes an increase in the excess pore water pressure.

\section{References}

[1] P. Lunardi, Design and Construction of Tunnels-Analysis of Controlled Deformation in Rocks and Soils, ADECO-RS, Springer, Berlin, Germany, 2008, Translation by B. A. James Davis (hons).

[2] S. Varadakos, Distinct element of the shimizu tunnel No. 3 in Japan, M.S. thesis, Virginia Polytechnic, Blacksburg, Va, USA, 2003.

[3] E. H. Davis, M. J. Gunn, R. J. Mair, and H. N. Seneviratne, "Stability of shallow tunnels and underground openings in cohesive material," Geotechnique, vol. 30, no. 4, pp. 397-416, 1980.

[4] R. J. Mair, Centrifugal modeling of tunnel construction in soft clay, Ph.D. thesis, University of Cambridge, Cambridge, UK, 1979.

[5] A. G. Bloodworth, Three-dimensional analysis of tunneling effects on structures to develop design methods, Ph.D. thesis, University of Oxford, 2002.

[6] C. Sagaseta, "Analysis of undrained soil deformation due to ground loss," Geotechnique, vol. 37, no. 3, pp. 301-320, 1987.

[7] L. Chow, Prediction of surface settlement due to tunneling in soft ground, M.S. thesis, University of Oxford, 1994.

[8] J. P. Carter, "Semi-analytical solution for swelling around a borehole," International Journal for Numerical and Analytical Methods in Geomechanics, vol. 12, no. 2, pp. 197-212, 1988.

[9] R. J. Mair and R. N. Taylor, "Prediction of clay behavior around tunnels using elasticity solutions," in Proceedings of Predictive Soil Mechanics Worth Memorial Symposium, pp. 449-463, Oxford, UK, 1993.
[10] A. Verruijt and J. R. Booker, "Surface settlements due to deformation of a tunnel in an elastic half plane," Geotechnique, vol. 46, no. 4, pp. 753-756, 1996.

[11] W. I. Chou and A. Bobet, "Predictions of ground deformations in shallow tunnels in clay," Tunnelling and Underground Space Technology, vol. 17, no. 1, pp. 3-19, 2002.

[12] R. B. Peck, "Deep Excavations and Tunneling in Soft Ground," in Proceeding of the 7th International Conference on Soil Mechanics and Foundation Engineering, pp. 225-290, Mexico City, Mexico, 1969.

[13] W. Rankin, "Ground movements resulting from urban tunneling prediction and effects," in Proceedings of the 23rd Conference of the Engineering Group of the Geological Society, pp. 79-92, London Geological Society, 1988.

[14] D. M. Potts and L. Zdravkovic, Finite Element Analysis in Geotechnical Engineering Application, Thomas Telford, 2001.

[15] A. M. Britto and M. J. Gunn, Critical State Soil Mechanics via Finite Elements, John Wiely \& Sons, New York, NY, USA, 1987.

[16] NCCLR, "Subsoil investigation for Baghdad metro line," Tech. Rep., National Center of Construction Laboratories and Research, Baghdad, Iraq, 1986.

[17] W. Zhu, S. Li, S. Li, W. Chen, and C. F. Lee, "Systematic numerical simulation of rock tunnel stability considering different rock conditions and construction effects," Tunnelling and Underground Space Technology, vol. 18, no. 5, pp. 531-536, 2003.

[18] K. M. Lee and R. K. Rowe, "Deformations caused by surface loading and tunnelling: the role of elastic anisotropy," Geotechnique, vol. 39, no. 1, pp. 125-140, 1989.

[19] K. Y. Lo and R. K. Rowe, "Prediction of ground subsidence due to tunneling in clays," Tech. Rep. GEOT-10-82, University of Western Ontario, Ontario, Canada, 1982.

[20] R. M. C. Ng and K. Y. Lo, "The measurements of soil parameters relevant to tunnelling in clays," Canadian Geotechnical Journal, vol. 22, no. 3, pp. 375-391, 1985.

[21] T. I. Addenbrook, Numerical analysis of tunneling in stiff clay, Ph.D. thesis, Imperial College, University of London, 1996.

[22] P. F. M. Guedes and S. C. Pereira, "The role of the soil $\mathrm{k}_{0}$ value in numerical analysis of shallow tunnels," in Proceeding of the International Symposium on Geotechnical Aspects of Underground Construction in Soft Ground, pp. 379-384, Balkema, Rotterdam, The Netherlands, 2000.

[23] M. Dolezalova, "Approaches to numerical modeling of ground movements due to shallow tunneling," Soil Interaction in Urban Civil Engineering, vol. 2, pp. 365-373, 2002.

[24] G. T. K. Lee and C. W. W. Ng, "Three-dimensional analysis of ground settlements due to tunneling: role of $\mathrm{k}_{0}$ and stiffness anisotropy," in Proceeding of the International Symposium on Geotechnical Aspects of Underground Construction in Soft Ground, pp. 617-622, Specifique, Lyon, France, 2002.

[25] T. I. Addenbrooke, D. M. Potts, and A. M. Puzrin, "The influence of pre-failure soil stiffness on the numerical analysis of tunnel construction," Geotechnique, vol. 47, no. 3, pp. 693$712,1997$. 

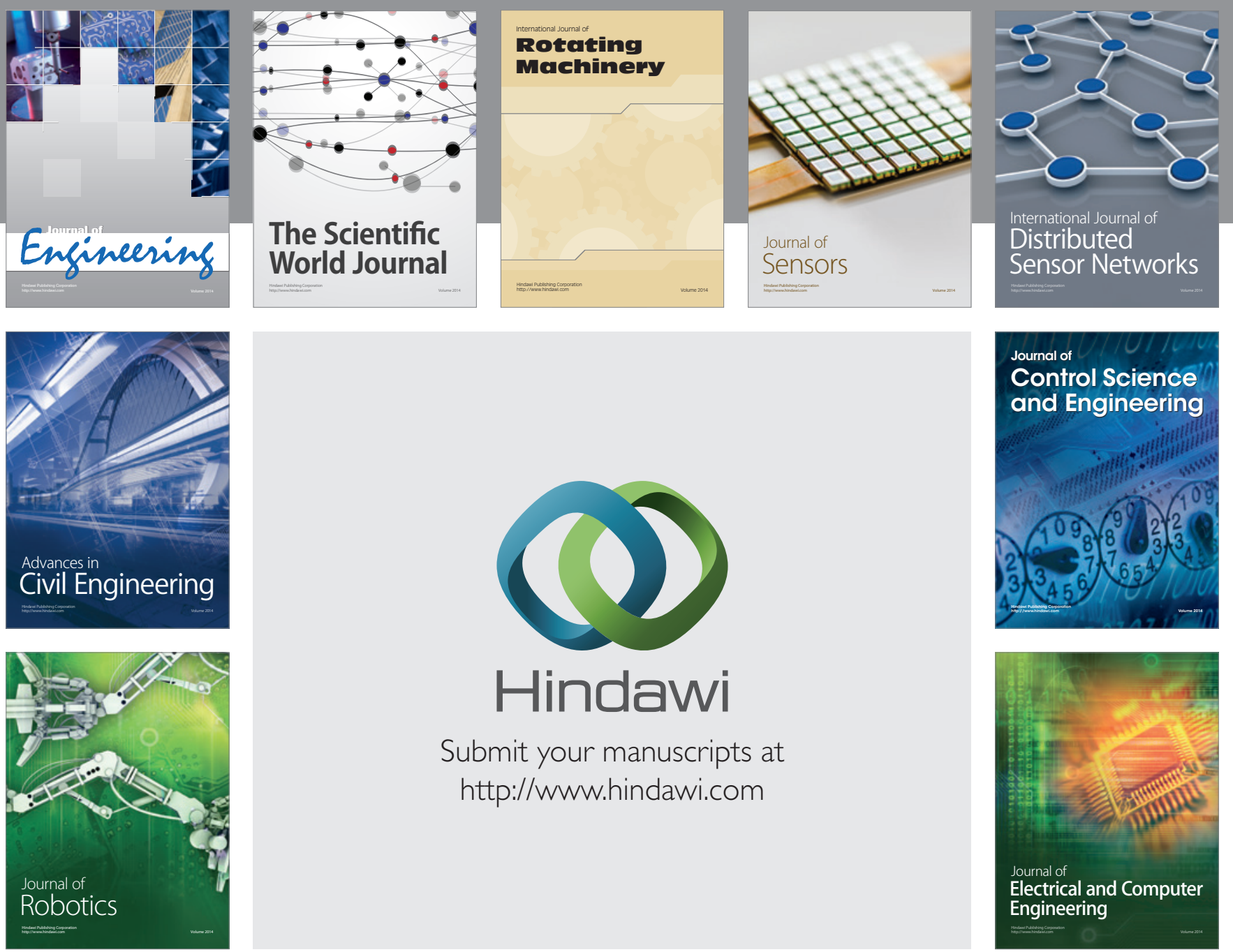

Submit your manuscripts at

http://www.hindawi.com
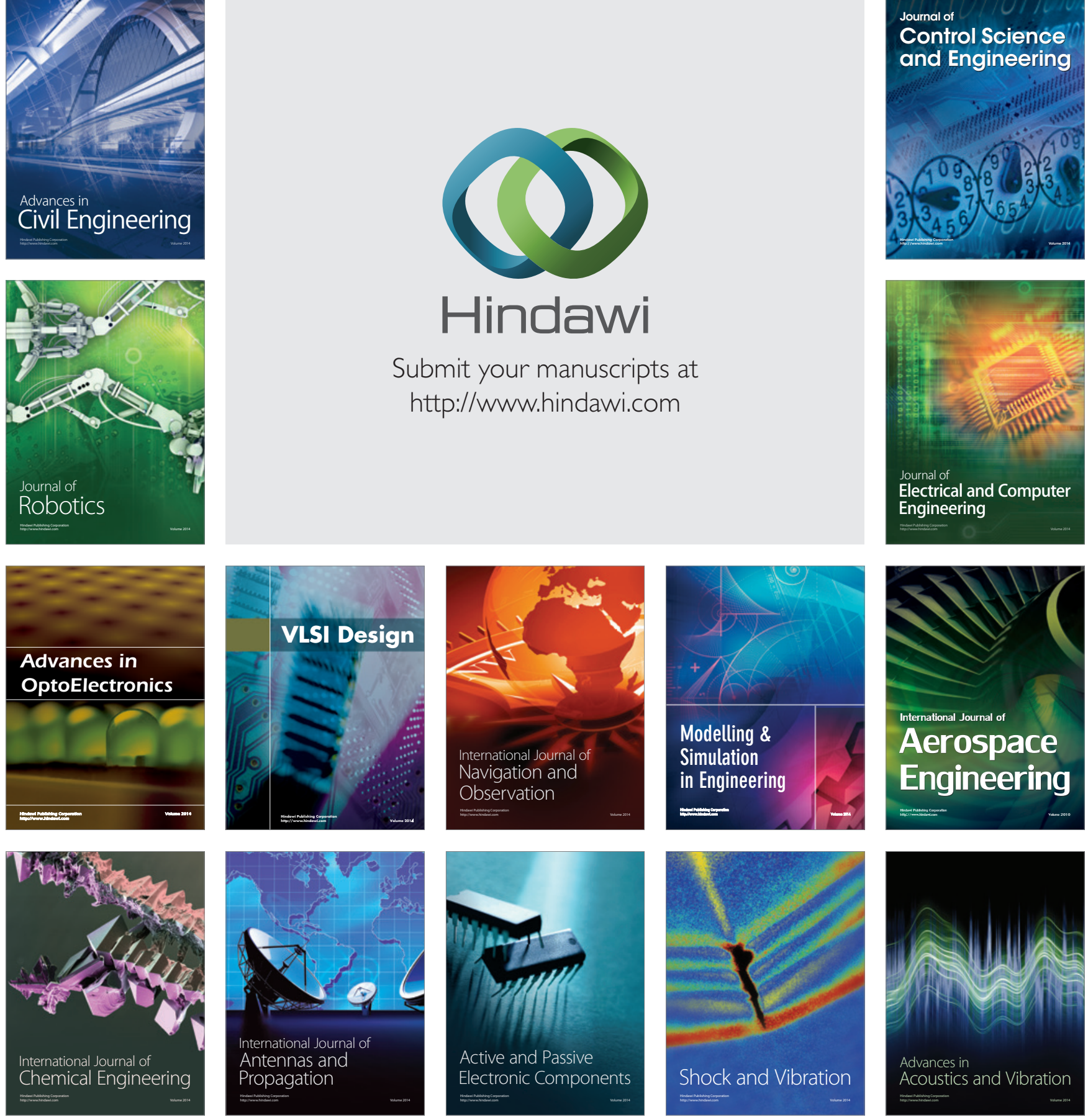